\title{
Micromagnetic simulation of the magnetic exchange spring system $\mathrm{DyFe}_{2} / \mathrm{YFe}_{2}$
}

\author{
Jürgen P. Zimmermann, ${ }^{\text {a) }}$ Giuliano Bordignon, Richard P. Boardman, Thomas Fischbacher, \\ and Hans Fangohr \\ Computational Engineering and Design Group, School of Engineering Sciences, University of Southampton, \\ Southampton SO17 1BJ, United Kingdom
}

Kevin N. Martin, Graham J. Bowden, Alexander A. Zhukov, and Peter A. J. de Groot

School of Physics and Astronomy, University of Southampton, Southampton SO17 1BJ, United Kingdom

(Presented on 31 October 2005; published online 25 April 2006)

\begin{abstract}
Magnetic measurements of [110] [50 $\left.\mathrm{A} \mathrm{DyFe}_{2} / 200 \AA \mathrm{YFe}_{2}\right]$ reveal a rich switching behavior: the formation of exchange springs in this system of alternating hard and soft layers can be observed for low temperatures (LTs). For high temperatures (HTs), the appearance of the hysteresis loop changes significantly, implying a more complicated reversal process. In this article, we reproduce hysteresis loops for net and compound-specific magnetizations by means of micromagnetic simulations and assess the quality by a direct comparison to recent x-ray magnetic circular dichroism measurements. The HT switching characteristics, showing a magnetization reversal of the hard magnetic layer before the soft magnetic layer, are investigated and understood on the basis of detailed magnetic configuration plots. The crossover of LT to HT switching patterns is explained by energy considerations, and the dependence on different parameters is outlined. (C) 2006 American Institute of Physics. [DOI: 10.1063/1.2172560]
\end{abstract}

Magnetic exchange spring systems are multilayers or composites of magnetically hard and soft material that are exchange coupled across their interfaces. A common aspect is the pinning of the magnetization of the hard magnetic material to an anisotropy minimum, where the applied field winds the magnetization of the soft material out of an equilibrium direction opposite the hard magnetization direction. This resembles a mechanical torsion spring, thus the name "exchange spring." materials ranges from applications as permanent magnets, ${ }^{3}$ as giant magnetoresistance (GMR) spin devices, ${ }^{4}$ to usage for advanced recording media. ${ }^{5}$

The system studied in this article is a model of a [110] $\left[50 \AA \mathrm{DyFe}_{2} / 200 \AA \mathrm{YFe}_{2}\right] \times 27$ Laves phase superlattice. Macroscopic magnetization measurements as well as compound-resolved $\mathrm{x}$-ray magnetic circular dichroism (XMCD) analysis ${ }^{6,7}$ reveal an unexpected switching behavior with three irreversible switchings for high temperature (HTs) and a typical exchange spring magnetization reversal for low temperature (LTs). We investigate the spin configuration of this unexpected reversal behavior by means of micromagnetic simulations. The resulting compound-specific and net hysteresis loops are presented and compared with experimental data. ${ }^{6,7}$ Good qualitative agreement legitimates the simulation data and allows the interpretation of resolved configuration plots that clarify the different switching behavior.

$\mathrm{DyFe}_{2}$ is a ferrimagnet, and for the computational modeling we assume a rigid ferromagnetic $\mathrm{Fe}-\mathrm{Fe}$ and antiferromagnetic Fe-Dy coupling inside a crystal cell [see Fig. 1(a)]. This allows us to calculate a net magnetization of $\mathrm{DyFe}_{2}$ by subtracting the magnetization of $\mathrm{Fe}$ from that of Dy. The

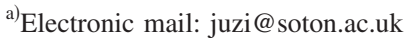

magnetization of $\mathrm{Y}$ is negligible, and $\mathrm{Fe}$ solely contributes to the net magnetization of $\mathrm{YFe}_{2}$. With increasing temperature, the magnetic moment of Dy diminishes faster than that of $\mathrm{Fe}$, and $\mathrm{DyFe}_{2}$ and $\mathrm{YFe}_{2}$ magnetizations tend to level [insert of Fig. 2(a)]. The $\mathrm{Fe}$ atoms are ferromagnetically coupled, effecting positive intralayer and negative across-layer exchange couplings (coupling constants $A_{\text {intra }}=-A_{\text {across }}$ $=1.46 \times 10^{-11} \mathrm{~J} / \mathrm{m}$ ) between the net moments.

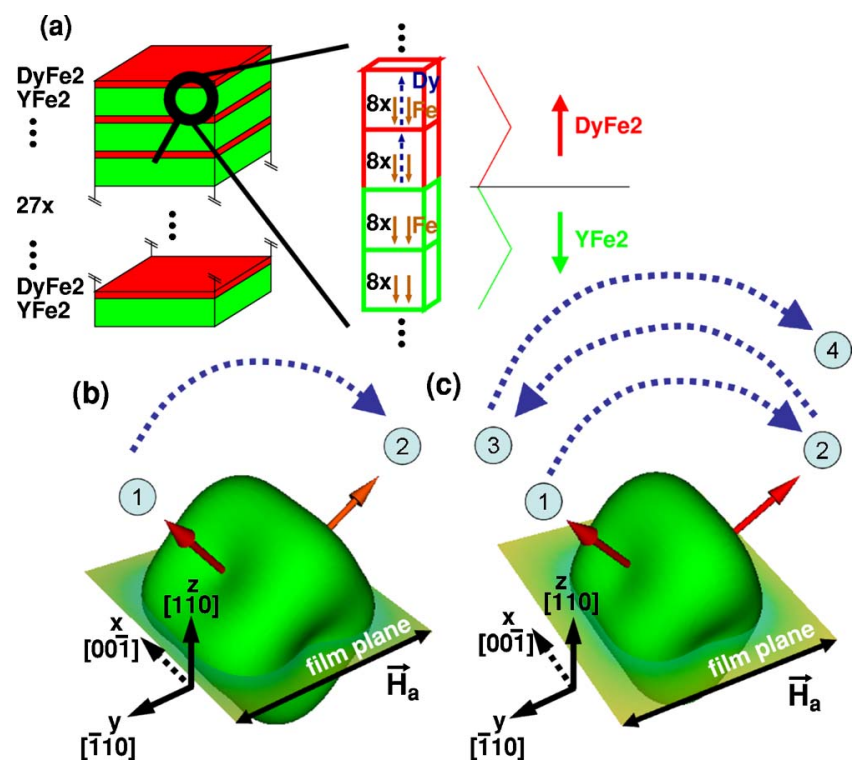

FIG. 1. (Color online) (a) Sketch of the compound layers and a close-up showing the atomic spin coupling. Normalized anisotropy energy surface (green) and average net magnetization directions (red) for the $\mathrm{DyFe}_{2}$ layers for $200 \mathrm{~K}$ (b) and $350 \mathrm{~K}$ (c). The dotted lines mark the irreversible switchings under a varied applied field. The spin states of the $\mathrm{DyFe}_{2}$ layers are indicated by the circled numbers. 

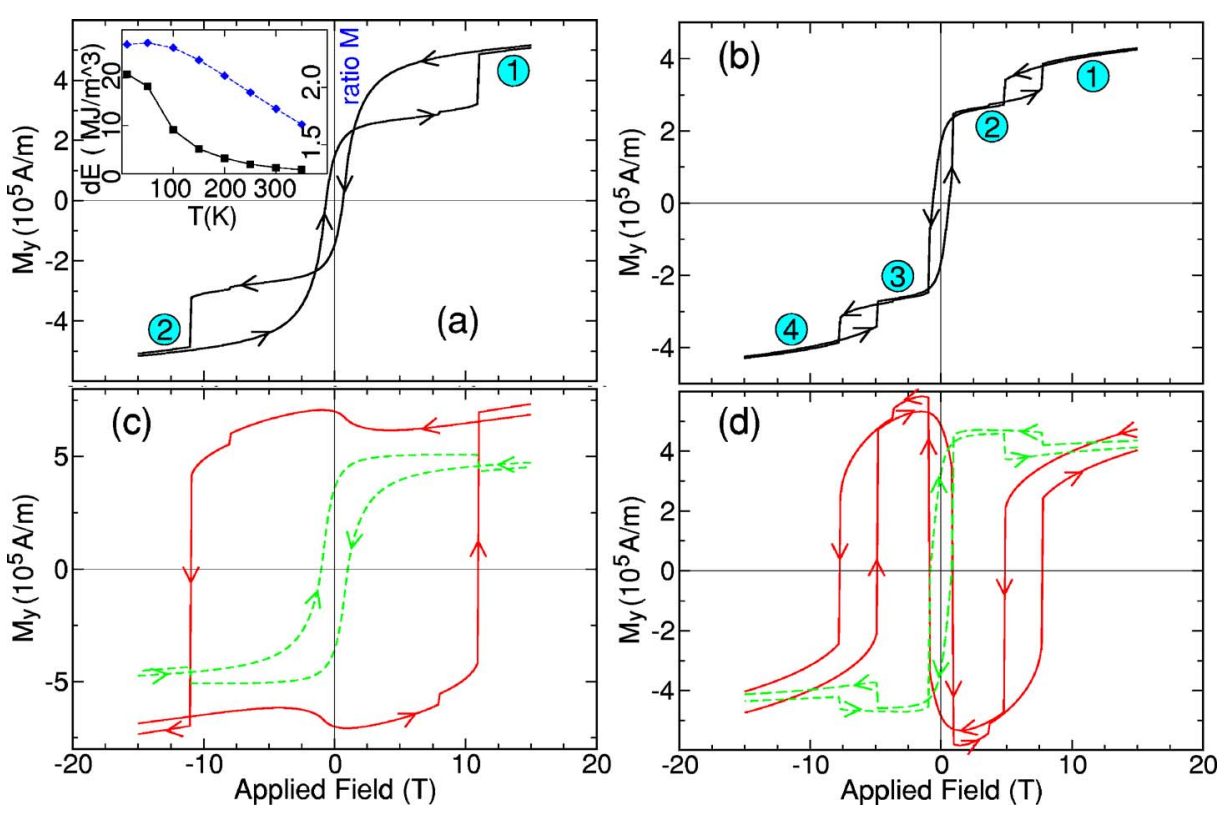

FIG. 2. (Color online) Hysteresis loops of the net [(a) and (b)] and compound-specific [(c)and (d)] magnetizations $M_{y}$ for $200 \mathrm{~K}$ (left) and $350 \mathrm{~K}$ (right). The solid (red) lines indicate the $y$ magnetization of the $\mathrm{DyFe}_{2}$ layers and the dashed (green) lines of the $\mathrm{YFe}_{2}$ layers in (c) and (d). The circled numbers in (a) and (b) correspond to the magnetization states shown in Fig. 1. For $350 \mathrm{~K}$, spin configurations for states $1-3$ are depicted in Fig. 3. (Inset) The anisotropy energy barrier $d E\left(\mathrm{MJ} / \mathrm{m}^{3}\right.$, black squares) and the ratio of the net magnetization of $\mathrm{DyFe}_{2}$ to $\mathrm{YFe}_{2}$ (blue diamonds) for various temperatures $T$. The lines are a guide to the eye.
While we can ignore magnetic anisotropy for $\mathrm{YFe}_{2}$, anisotropy of $\mathrm{DyFe}_{2}$ plays a crucial role in the understanding of the switching behavior, bearing a cubic magnetocrystalline (MC) contribution of the bulk Dy with easy axes in $\langle 100\rangle$ and a strain term due to epitaxial effects. The MC anisotropy values are extracted from Ref. 8. The strain term gets increasingly important for temperatures above $170 \mathrm{~K}$, when it gradually rotates the easy axis in the (001) plane towards [110]. Strain parameters used in the simulations are derived from a single-ion point charge model in qualitative agreement with Ref. 9. In Fig. 1, the overall anisotropy shapes are depicted for $200 \mathrm{~K}$ (b) and $350 \mathrm{~K}$ (c). The magnetocrystalline anisotropy contributions fade comparably slowly for Dy (compared to other rare-earth materials ${ }^{8}$ ) with raising temperature, and the cubic shape is pronounced for both temperatures. However, for $350 \mathrm{~K}$, the cubic symmetry is notably distorted towards the $z$ direction, favoring directions in the film plane. The insert of Fig. 2(a) shows the calculated values of the anisotropy energy barrier in the $z$ direction separating the easy axes, indicating a rapid decay of the barrier with raising temperature.

We use the OOMMF code ${ }^{10}$ to perform the micromagnetic simulations. The layer structure and the direction of the applied field $\mathbf{H}_{\mathrm{a}} \|[\overline{1} 10]$ correspond to that of Refs. 6 and 7 . A cell size of $1 \mathrm{~nm}^{3}$ provides sufficient resolution with respect to an exchange length of $3.4 \mathrm{~nm}$. For computational feasibility, the magnetization of each $x y$ layer is represented by one cell. The simulations account for thermal effects by temperature-dependent magnetization and anisotropy values. Demagnetizing effects are considered within the constraint of limited $x y$ resolution.

The simulations were performed for various temperatures $T$. The resulting switching patterns can be divided into a LT regime for $T \leqslant 300 \mathrm{~K}$ and a HT regime for $T>300 \mathrm{~K}$. Results for each regime are depicted in Fig. 2, comprising of net and compound-specific hysteresis loops. Starting off with high $\mathbf{H}_{\mathrm{a}}$ and almost saturated magnetization, the net hysteresis loop for $200 \mathrm{~K}$ (a) shows an accelerated decline of the magnetization, with a negative coercivity of $0.65 \mathrm{~T}$. At roughly zero $\mathbf{H}_{\mathrm{a}}$, the magnetization curve turns almost flat. These are the characteristics of an exchange spring system: with decreasing applied field, the magnetically soft layers unwind until the system reaches an ordered ferrimagnetic state. For $H_{\mathrm{a}} \leqslant-11 \mathrm{~T}$, magnetization drops to almost negative saturation when the hard layers irreversibly switch into the direction of the field. This is underlined by the compound-specific hysteresis loop [Fig. 2(c)], showing the soft $\mathrm{YFe}_{2}$ magnetization $\mathbf{M}_{\mathrm{YFe}_{2}}$ forming an exchange spring first at a small positive field and the hard $\mathrm{DyFe}_{2}$ magnetization $\mathbf{M}_{\mathrm{DyFe}_{2}}$ switching subsequently at a high reversed $\mathbf{H}_{\mathrm{a}}$.

For $350 \mathrm{~K}$, the appearance of the hysteresis loop has changed drastically: The net hysteresis loop (b) shows a first irreversible step at $H_{\mathrm{a}} \approx+5 \mathrm{~T}\left(\Delta M \approx 0.7 \times 10^{5} \mathrm{~A} / \mathrm{m}\right)$. Coercivity is positive $(0.65 \mathrm{~T})$, accompanied by a second irreversible step of $\Delta M \approx 5 \times 10^{5} \mathrm{~A} / \mathrm{m}$. A third irreversible step is at $-7.6 \mathrm{~T}$, with a similar appearance as the first step. The compound-specific hysteresis loop (d) gives further insight: it is the magnetically hard $\mathrm{DyFe}_{2}$ layer to switch first into a direction opposing $\mathbf{H}_{\mathrm{a}}$. Both $\mathbf{M}_{\mathrm{DyFe}_{2}}$ and $\mathbf{M}_{\mathrm{YFe}_{2}}$ reverse at the second step, and $\mathbf{M}_{\mathrm{DyFe}_{2}}$ finally snaps towards $\mathbf{H}_{\mathrm{a}}$ at the third step. A vanishing $x$ magnetization component for all simulations indicates that the reversal processes take place in the (001) plane.

For both 200 and $350 \mathrm{~K}$, the sequence of irreversible switchings is outlined by the average $\mathbf{M}_{\mathrm{DyFe}_{2}}$ in Fig. 1, represented by the arrows superimposed on the anisotropy surfaces. It rests in the energy minimum between the [010] and [110] directions or the equivalent $z x$ mirrored energy minimum, and is slightly elongated from this position by the applied field and the exchange interaction of the $\mathbf{M}_{\mathrm{YFe}_{2}}$. For $200 \mathrm{~K}$, the average $\mathbf{M}_{\mathrm{DyFe}_{2}}$ switches once, and for $350 \mathrm{~K}$ three times.

The hysteresis loops achieved by micromagnetic simulations agree with the experimental hysteresis loops (Fig. 1 of Ref. 6) in the existence of distinct switching patterns for LT 


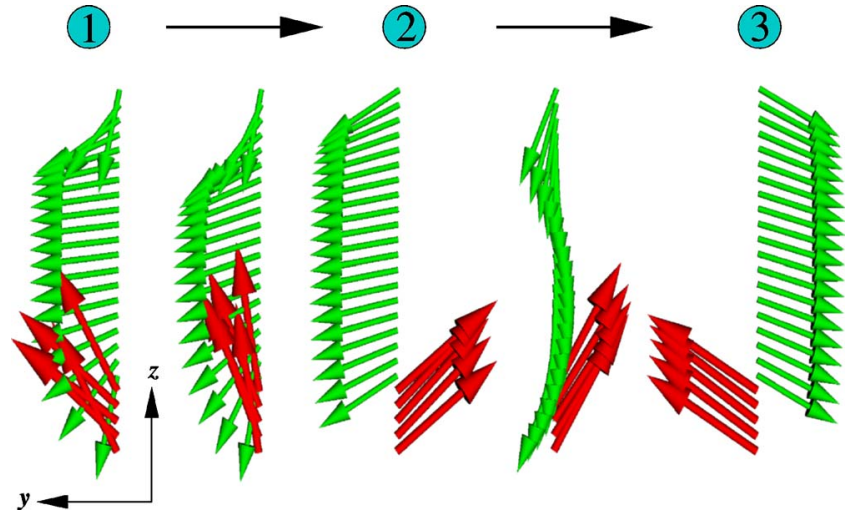

FIG. 3. (Color online) Spin configurations of an inward double layer for 350 $\mathrm{K}$ for states $1-3$ as depicted in Fig. 2, and their transition states. The applied field points along the $\pm y$ direction. The $\mathrm{DyFe}_{2}$ layer (red arrows) is located below the $\mathrm{YFe}_{2}$ layer (green arrows), where the arrows indicate the net magnetization vector per computational cell of $1 \mathrm{~nm}^{3}$.

and HT, the sequence of the reversals of each compound as well as the relative magnetization changes involved, and the sign and approximate value of the coercive field. Quantitative differences comprise of higher switching fields and a shift of the crossover point between the LT and HT regime towards higher temperatures. This can be accounted to the inability of the model to form complex magnetic structures in the $x y$ plane and to the underestimation of demagnetizing effects. However, the qualitative conformity of the hysteresis loops legitimates a further analysis of the simulation data for a better understanding of the interesting HT switching behavior.

In Fig. 3, the HT spin configurations are visualized with the resolution of the finite difference grid $(1 \mathrm{~nm})$. Note that the magnetization vectors do not rotate out of the $y z$ plane, the plane of the graph. For high positive applied field (state 1), $\mathbf{M}_{\mathrm{YFe}_{2}}$ is mostly aligned with $\mathbf{H}_{\mathrm{a}}$ (+y direction). $\mathbf{M}_{\mathrm{DyFe}_{2}}$ is settled in the anisotropy dip close to [010], towards $\mathbf{H}_{\mathrm{a}}$, but frustrating the exchange energy between the layers, giving a tight exchange spring. When $\mathbf{H}_{\mathrm{a}}$ is gradually reduced $(1 \rightarrow 2)$, the influence of exchange energy catches up, turning $\mathbf{M}_{\mathrm{DyFe}_{2}}$ towards a direction opposing $\mathbf{M}_{\mathrm{YFe}_{2}}$ that is still mostly aligned with $\mathbf{H}_{\mathrm{a}}$. Next, $\mathbf{M}_{\mathrm{DyFe}_{2}}$ overcomes the anisotropy energy barrier and flips into the minimum close to [100] (state 2): first irreversible step. In remanence state, $\mathbf{M}_{\mathrm{DyFe}_{2}}$ rests in the latter anisotropy minimum, and the exchange energy keeps $\mathbf{M}_{\mathrm{YFe}_{2}}$ in an opposing direction. Since the dominating $\mathbf{M}_{\mathrm{YFe}_{2}}$ vectors are still pointing in the direction of $\mathbf{H}_{\mathrm{a}}$, the coercivity is positive. With $\mathbf{H}_{\mathrm{a}}$ reversing $(2 \rightarrow 3), \mathbf{M}_{\mathrm{YFe}_{2}}$ gradually rotates towards the $-y$ direction. However, $\mathbf{M}_{\mathrm{DyFe}_{2}}$ is still locked in the anisotropy dip, and exchange interaction keeps $\mathbf{M}_{\mathrm{YFe}_{2}}$ from aligning with the new field direction. Consequently, a large interface wall is formed. The torque applied by the increasing reversed $\mathbf{H}_{\mathrm{a}}$ acts on $\mathbf{M}_{\mathrm{YFe}_{2}}$, which in turn drags $\mathbf{M}_{\mathrm{DyFe}_{2}}$ back over the anisotropy energy barrier via exchange interaction. $\mathbf{M}_{\mathrm{DyFe}_{2}}$ returns to the anisotropy minimum close to [010] and allows $\mathbf{M}_{\mathrm{YFe}_{2}}$ to further rotate towards $\mathbf{H}_{\mathrm{a}}$. This is reflected in the second irreversible step to state 3 , with a high magnetization amplitude due to the synchronous reversal of both compounds. With further increasing negative applied field, another switching occurs, based on the same mechanism as the first switching $(1 \rightarrow 2)$.

The reason for the existence of the observed HT reversal process is apparent in the temperature dependence of the anisotropy barrier and the ratio of the net magnetization of the two compounds [insert of Fig. 2(a)]: For HTs, the anisotropy barrier becomes sufficiently low to allow an isolated switching of $\mathbf{M}_{\mathrm{DyFe}_{2}}$. Additionally, with the growing magnetic $\mathrm{YFe}_{2}$ dominance for increasing temperatures, $\mathbf{M}_{\mathrm{YFe}_{2}}$ stays closely aligned with $\mathbf{H}_{\mathrm{a}}$, and the exchange spring is formed by the winding of $\mathbf{M}_{\mathrm{DyFe}_{2}}$.

In summary, micromagnetic modeling of $\mathrm{DyFe}_{2} / \mathrm{YFe}_{2}$ exchange spring systems has proven its ability to reproduce hysteresis loops with characteristics matching those of experimental work. It enabled a thorough analysis of the HT switching behavior, clarifying the unexpected magnetic reversal mechanism, with the hard $\mathrm{DyFe}_{2}$ layers switching sooner than the soft $\mathrm{YFe}_{2}$ layers. The strong thermal dependence of anisotropy plays a key role for the reversal processes, and the interplay with exchange and Zeeman energy is understood on a qualitative level. The successful simulation of the $\mathrm{DyFe}_{2} / \mathrm{YFe}_{2}$ system allows us to proceed to other rare-earth-iron compounds, including $\mathrm{ErFe}_{2}$ and $\mathrm{TbFe}_{2}$, with different anisotropies, and switching patterns which yet need to be explained.

\footnotetext{
${ }^{1}$ M. Sawicki, G. J. Bowden, P. A. J. de Groot, B. D. Rainford, R. C. C. Ward, and M. R. Wells, J. Appl. Phys. 87, 6839 (2000).

${ }^{2}$ E. E. Fullerton, J. S. Jiang, M. Grimsditch, C. H. Sowers, and S. D. Bader, Phys. Rev. B 58, 12193 (1998).

${ }^{3}$ E. F. Kneller and R. Hawig, IEEE Trans. Magn. 27, 3588 (1991).

${ }^{4}$ S. N. Gordeev, J.-M. L. Beaujour, G. J. Bowden, P. A. J. de Groot, B. D. Rainford, R. C. C. Ward, M. R. Wells, and A. G. M. Jansen, Phys. Rev. Lett. 87, 186808 (2001).

${ }^{5}$ T. Ando and T. Nishihara, IEEE Trans. Magn. 33, 2983 (1997).

${ }^{6}$ K. Dumesnil, C. Dufour, P. Mangin, F. Wilhelm, and A. Rogalev, J. Appl. Phys. 95, 6843 (2004)

${ }^{7}$ K. Dumesnil, C. Dufour, P. Mangin, A. Rogalev, and F. Wilhelm, J. Phys.: Condens. Matter 17, 215 (2005).

${ }^{8}$ U. Atzmony and M. P. Dariel, Phys. Rev. B 13, 4006 (1976).

${ }^{9}$ A. Mougin, C. Dufour, K. Dumesnil, and P. Mangin, Phys. Rev. B 62, 9517 (2000)

${ }^{10}$ M. J. Donahue and D. G. Porter, OOMMF User's Guide (National Institute of Standards and Technology, Gaithersburg, MD, 1999; interagency Report NISTIR 6376.
} 